\title{
The Double-Pandemic: COVID-19 and Seasonal Influenza during Winter 2020-2021 is an Expected Surprise
}

\section{Rapaport $\mathrm{C}^{1,2}$ and Ashkenazi I ${ }^{1,3 *}$}

${ }^{1}$ Institute for Regulation of Emergency and Disaster, College of Law and Business, Israel ${ }^{2}$ Department of Geography and Environmental Studies, University of Haifa

${ }^{3}$ Faculty of Health Sciences, Ben Gurion University of the Negev, Israel

*Corresponding author: Prof. Isaac Ashkenazi, Faculty of Health Sciences, Ben Gurion University of the Negev and Institute for Regulation of Emergency and Disaster, College of Law and Business, Israel, Tel: +972-54-5424605; Email: Isaac_Ashkenazi@ksg01.harvard.edu

\section{Perspective \\ Volume 4 Issue 2}

Received Date: November 16, 2020

Published Date: December 04, 2020

DOI: $10.23880 /$ phoa-16000166

\section{Abstract}

Given COVID-19 pandemic and seasonal influenza in winter 2020-2021 we describe the challenges, both medical and social, posed by the potential double-pandemic of diseases. We also provide practical strategies to cope with this double-pandemic.

Keywords: Vaccinations; COVID-19; Seasonal Influenza; Population; Public trust; Public Behavior; Leadership; Pandemic Fatigue

\section{Background}

The COVID-19 pandemic has proven to be a highlycontagious, lethal, and asymmetrical disease [1] that attacks mainly older people, and spreads rapidly through social gatherings and in closed places. So far, two factors have shown to play critical roles in stemming the spread of the pandemic: the public's behavior (especially adherence to the instructions - wearing face masks, keeping physical distance from others and hygiene) and decision makers' management of the outbreaks such as shutting down or reopening the education systems, public transportation and the economic sectors. During the upcoming months, the COVID-19 pandemic will continue to spread in parallel with the seasonal influenza in the northern hemisphere. We review challenges that might unfold give this potential double pandemic, while considering strategies to prepare and mitigate its effect on both the public health and population behavior domains. Findings from Australia and New Zealand (who have already experienced the 2020 winter season) show a decrease in seasonal flu rates ${ }^{1}$. This

1 https://www1.health.gov.au/internet/main/publishing.nsf/Con tent/7938AA11F1D2ABBDCA25860A0007C1BF/\$File/flu-14-2020.pdf is probably due to the population's adherence to protection measures against COVID-19, extensive testing, higher levels of vaccinations ${ }^{2}$, closure of the educational system and low global air traffic into the countries. Nevertheless, it is known that during the year, antigenic changes in influenza viruses might lead to different prevailing viral strains in the southern and northern hemispheres. As a result, not much can be learned from the Australian winter case for what is expected in the northern hemisphere. The concern in many countries is of a major outbreak with a serious potential for double-pandemic: COVID-19 and seasonal influenza. Such a double pandemic can have both short and long terms effects in various domains: health, society, economy, education, trust in governments and leadership. Given the worrying starting-point with high numbers of positive cases of COVID- $19^{3}$ (which was not the case in Australia and in other

2 https://www.canberratimes.com.au/story/6756635/hereswhy-you-have-to-wait-for-a-flu-shot/

3 Australia reported 4 cases per 100,000 population at the beginning of its winter between June 22nd-July 5th, 2020. Source: https://www1. health.gov.au/internet/main/publishing.nsf/Content/1D03BCB527F40C8 BCA258503000302EB/\$File/covid_19_australia_epidemiology_report_21_ fortnightly_reporting_period_ending_19_july_2020.pdf). This in comparison with much higher levels in Europe and the US as of the beginning of October, 
countries in the southern hemisphere), countries in the Northern hemisphere should consider their preparedness and mitigation actions towards this double pandemic.

\section{The Challenges of the Double Pandemic}

\section{Prevention}

Personal Protection Measures: The protection instructions - wearing face masks and maintaining physical distance as well as personal hygiene - was found to be effective for minimizing risk of infection for COVID-19. Given that the world's population is familiar with these instructions and is following them, decision makers should also recommend using it against the seasonal flu and other respiratory diseases in general. This highlights the question, however, of whether such a recommendation is feasible when there is no immediate or direct threat such as an active pandemic.

Vaccination Rates: Countries are encouraging influenza vaccinations to reduce infections rates and complications associated with seasonal influenza outbreaks, and public demand for vaccines is expected to be higher this year. However, challenges could include shortages in supplies as well as socioeconomic-based inequality of access, which could deprive those who would be unable to privately purchase the vaccine.

Vaccines' effectiveness: According to the $C D C$, influenza vaccines are more effective against influenza $B$ and $A(H 1 N 1)$ than for influenza $A(H 3 N 2)^{4}$. Furthermore, the vaccine "reduces the risk of flu illness by between $40 \%$ and $60 \%$ among the overall population during seasons when most circulating flu viruses are well-matched to the flu vaccine" (CDC, 20205). Given this, there is high importance and urgency to prepare national and local healthcare systems for the double pandemic, as well as workers in critical positions. This insight raises an important question: should the vaccination be mandatory for all of the population? (the CDC recommends the vaccine to every person aged 6 months and $u^{6}$.)

Innovative fast, available and approachable testing: local testing systems that provide fast and reliable results, that are available in every clinic or public place, and that are accessible

2020 (Oct.16TH): 312 cases per 100,000 population in Spain, 346 cases per 100,000 population in France, 65 cases per 100,000 population in Germany and 2,456 cases per 100,000 population in the USA (Sources: https://covid. cdc.gov/covid-data-tracker/\#trends_totalandratecases; https://www. ecdc.europa.eu/en/publications-data/download-todays-data-geographicdistribution-covid-19-cases-worldwide .

4 https://www.cdc.gov/flu/vaccines-work/vaccineeffect.htm

5 https://www.cdc.gov/flu/vaccines-work/vaccineeffect.htm

6 https://www.cdc.gov/flu/prevent/whoshouldvax.html to every person will help to improve the management of the double pandemic. The advantages of such testing systems lie in two desired outcomes: they will improve the treatment for positive cases (to prevent outbreak acceleration) and they will allow adequate medical care (which will reduce mortality).

\section{Response and Pandemic Management}

Critical Scarce Resources: Both diseases, and each on its own, might put health systems, and hospitals in particular, under extreme threat as the care and medical treatment for both diseases are based on the same scarce resources: separated hospitalization rooms, ventilators, skilled personnel and personal protection equipment.

Additive Effect: The combination of two contagious respiratory diseases, one of which (COVID-19) can also be asymptomatic, might put extreme pressure on the ability of healthcare systems to function properly. Furthermore, a person might get infected with both diseases, which further increases the risk for both the population and the healthcare systems.

Diagnostics and testing: upper respiratory tract infections might symptomize as COVID-19 including ILI (influenza-like illness) such as rhinovirus ("common cold"), respiratory syncytial virus (RSV) as well as influenza and pneumonia. The care for ILI burdens both community clinics and hospitals. For example, 60 million patient visits were recorded in the USA in the winter season of 2019-20207. Given the high prevalence of these illnesses, patients with mild symptoms might refer to testing even more than once in the season, which might lead to enormous pressure on the testing systems.

Laboratories: national and local laboratories will be extremely overwhelmed due to the need to test influenza, COVID-19 and other ILI (influenza-like illnesses). Nonetheless, results should be received as fast as possible in order to control the outbreak and increase cooperation with the patient.

Chains of Infections: Given the expected high levels of upper respiratory tract infections and testing, the epidemiological investigations in outbreak zones might experience an extreme burden with increased probability of mistakes and the potential for a total breakdown of the investigations' system.

Treatment and quarantine - the need for designated hospitalization facilities: The treatment for COVID-19 and influenza is different, leading to increased burden on

7 https://www.cdc.gov/flu/weekly/overview.htm 
hospitals' medical personnel, as well as increased risk of exposure to both diseases. National healthcare leaders should make decisions concerning how to prepare systematically for the response: should specific hospitals and/or healthcare facilities be designated to care for COVID-19 only? or for both diseases? Would this mean that other hospitals would provide care for all other medical needs? Another option that should be considered is designating triaging facilities, which would manage patients' flow after quick testing. Such separation is critical to avoid cross-infection and to ensure adequate treatment to the patients.

High-Risk Populations: The combination of both COVID-19 and influenza might put certain populations- such as the elderly- at an even higher risk. Moreover, the combination of both diseases might lead some populations, who are not at high risk for COVID-19 but are at high risk for influenza- such as young children- to increased need for hospitalization.

\section{Course of the Diseases}

Transmission: While influenza is known to be airborne transmitted, COVID-19 might also be transmitted by aerosols. This increases the chances of infection even when people gather outdoors. This demands using other protection measures, for example, wearing face masks even in open areas and maintaining wider physical distances from one another.

Influenza is not asymmetric: COVID-19 is an asymmetric disease (Rapaport \& Ashkenazi, 2020) which attacks mostly the elderly. Influenza might risk and affect people of all ages and health conditions.

Progression of the Disease: In both cases, symptoms might present five days after the infection. In the case of influenza, however, the main illness might appear at an earlier stage after the infection, while COVID-19 develops later with longer effects of symptoms even after recovery. This might lead to a continuous burden on healthcare services and institutions.

Effect on Children: Unlike COVID-19, influenza does affect children, with possible complications especially in young children (younger than 5 years old) [2]. Additionally, children are critical vectors for transmitting influenza. The reopening of schools will lead to increased levels of influenza transmission rates, which might affect adults in the families and in the entire community.

\section{Population Behavior}

The Double Pandemic as a Psychosocial Problem: The population plays a critical role in mitigating outbreaks and demand for medical services. The combination of COVID-19 and seasonal influenza might increase stress and anxiety of getting infected leading to hospital surge for testing. In such case, countries will have to cope mainly with a psychosocial problem, besides the medical one. This might lead to lower levels of trust in the government and healthcare systems, and negative effects on compliance with instructions.

"Pandemic Fatigue" (WHO, 2020'): Given that COVID-19 has been a continuous situation, it might affect the population's ability to adhere to the protection instructions over time, as individuals typically become desensitized to the positive outcomes of complying with protection instructions (as well as the negative outcomes of noncompliance).

Need for Social Interactions after Lockdown: After each lockdown (be it national or local) people might intensively meet with their relatives or friends, which might, in turn, cause outbreaks.

Changes in Medical Services Consumption: As upper respiratory illnesses share similar symptoms, people might change their medical services consumption, which might accelerate worsening of current chronic illnesses. For example, people might avoid treatment at hospitals or community clinics because they are afraid of getting infected with COVID-19 or influenza, or do not want to burden the overloaded medical personnel. Furthermore, people who have symptoms of "common-cold" might visit the local clinic or emergency room (even more than once in the season) out of fear of being or getting infected by COVID-19 or influenza, and this may put increased burden on the already-stressed healthcare system.

Self-Isolation: The emotional and mental load might increase over time as well. As a result of fear and anxiety of getting infected, or by seeing pictures of overflowed hospitals, people might isolate themselves, or spontaneously quarantine themselves at home, which will have an enormous effect on the economy (which is basically open, but with little consumer traffic).

Social Exclusion: People might also denunciate those who show sickness symptoms in public, even if not related to any disease (for example, a smoker who spontaneously coughs). This could lead to social disruptions and clashes between groups.

\section{Suggested Coping Strategies}

Awareness of the challenges that such double pandemic

8 https://www.euro.who.int/en/media-centre/events / events/2020/10/pandemic-fatigue-reinvigorating-the-public-to-preventcovid-19 
might pose to national and local healthcare systems is important for tailoring an appropriate strategy given the risk factors, the population's characteristics and the coping employed so far. We suggest that preparedness for the double pandemic should include prevention, mitigation and communication with the public.

\section{Outbreak Prevention}

Decreasing COVID-19 Cases: The burden on the healthcare system should be lowered as much as possible before annual peaks of seasonal influenza.

Vaccinations for High-Risk Populations: Massive influenza vaccinations should be provided to local communities, while prioritizing high-risk populations, including healthcare workers, caregivers and workers in critical positions such as in infrastructure facilities (water, electricity, gas, and defense) and education systems (including both workers and students). The potential of cross-immunity, for COVID-19 via the flu vaccination [3], might lead to positive effects on both diseases - especially when also considering the positive effect of the protection measures taken by the public. In the case of high-risk populations, such as the elderly, or people who live in shelters, or populations who live in peripheral areas - vaccinations should be provided locally, with swift, affordable, and easy access.

Vaccination Availability: Given an expected increase in demand for flu vaccinations, countries should prepare in advance to be able to provide enough vaccinations to all population groups.

\section{Mitigation}

Surge Capacity: Increasing the capacities of hospitals and community clinics for patient intake surge (with the option to isolate COVID-19 patients, influenza patients, and those with both diseases) would enable better coping with both diseases.

Collaboration between States, Provinces and Areas: A national system should monitor the morbidity rates in different places in the country to allow efficient resource allocation and transfer of equipment, personnel and knowledge to enable better coping in places with extreme outbreaks.

Increased use of deliveries: Local businesses as well as local healthcare facilities should utilize available delivery services to provide services at homes for high-risk populations. This will decrease the need for high-risk populations to leave their houses, to enter crowded places and to risk exposure to pathogens. Deliveries are not restricted to groceries, cooked-food or medications; they can -and should- also include entertainment items (books, magazines, crafts and toys for young children), paying bills and other official documents, and treatment at home (such physiotherapy and occupational therapy). It should be noted that delivery people are prone to transmit viruses to clients, and therefore should be considered critical workers; they should therefore get vaccinated early and adhere closely to all protection instructions.

\section{Communication with the Public}

Intensive Communication: Should expose people to this new threat, focusing people's awareness on the possibility of getting infected with both diseases. The guiding line for the communication process should not be on rising fear, but rather on the community's shared goals and social dependence - those who feel symptoms should stay at the home. Parents who feel sick should consider whether to send their children to school or to keep them at home. Furthermore, given the psychosocial aspects of the doublepandemic, the terminology in risk communication should include appropriate terms such as 'keeping physical distance", rather than "keeping social distance".

Awareness of Preventing Infections in Public Places: Local communities' public institutions should raise awareness to the double pandemic threat. Schools, workplaces and public places should appoint a person or a team who will enforce physical distancing measures and face mask policies. Furthermore, school children might serve as excellent "ambassadors" of adherence to the instructions, while being exposed to the instructions at school and speaking about them at home with their parents.

Building Public Trust: Providing constant, simple, accurate, reliable and accessible information is critical for increasing public trust in the official outbreak management. Positive perceptions of leaders' decisions, as well as trust-building steps (such as constant communication in mass media at both the local and national levels), will increase the public's willingness to cooperate with the instructions while understanding the rationale behind far-reaching steps such as movement restrictions and business closures.

Finally, the combination of the two diseases in the winter season might raise the question of "what is the acceptable loss"? [4]. This means, finding the fragile balance between the loss of lives and keeping life routines while using lockdowns and reopening repeatedly, which-as described above- will have cascading effects on the public, their willingness to adhere to protection instructions, and their trust in local and national authorities. Preparing for the next stages of the COVID-19 pandemic management includes utilizing 
the vaccinations which will be available in the upcoming months. The horrifying scenario of the double pandemic raises a critical dilemma: on one hand, countries are willing to provide COVID-19 vaccinations for all citizens as soon as possible, while on the other hand, such vaccinations might not be fully approved yet, and their effectiveness or safety are not fully known. Countries' leaders and public health decision makers would have to decide: how to save more lives?

\section{References}

1. Rapaport C, Ashkenazi I (2020) Managing COVID-19:
Applying an Asymmetric Solution to an Asymmetric Problem. Journal of Emergency Medical Services.

2. Poehling KA, Edwards KM, Weinberg GA, Szilagyi P, Staat MA, et al. (2006) The underrecognized burden of influenza in young children. N Engl J Med 355(1): 31-40.

3. Debisarun PA, Struycken P, Dominguez AJ, Moorlag SJ, Taks E, et al. (2020) The effect of influenza vaccination on trained immunity: impact on COVID-19. medRxiv, pp: 22.

4. Ashkenazi I, Rapaport C (2020) Saving Lives Versus Saving Dollars: The Acceptable Loss for Coronavirus Disease 2019. Crit Care Med 48(8): 1243-1244. 